\title{
Georges Cuvier en España. La traducción de José Garriga y Baucís de Lecciones elementales de la historia natural de los animales
}

\author{
Georges Cuvier in Spain. José Garriga y Baucís's translation of \\ Lecciones elementales de la historia natural de los animales
}

\section{Matteo De Beni}

Università degli Studi di Verona. matteo.debeni@univr.it

Recibido: 30.04.2019. Aceptado: 10.08.2019

Resumen: El presente trabajo pretende analizar la versión española realizada por José Garriga y Baucís de Tableau élémentaire de l'histoire naturelle des animaux (17971798) de Georges Cuvier (1769-1832), promotor de la paleontología y de la anatomía comparada. La traducción en cuestión, publicada en 1834 en Valencia por el taller de Cabrerizo, es una muestra señera de la llegada a España de obras de Cuvier, entre cuyas contribuciones al desarrollo de la biología y la anatomía cabe precisamente la descripción -basada en criterios estructuralistas-del reino animal.

En el artículo se pone el foco tanto en aspectos que vinculan el texto estudiado con su "historia externa" -en particular, su intención didáctica en el cuadro de las disposiciones vigentes en la enseñanza reglada de la época- como en la confrontación entre la obra de Cuvier y su traducción española, lo cual permite evidenciar las peculiaridades de esta última con respecto al original y hacer hincapié en las elecciones léxicas y en la sensibilidad lingüística del traductor, quien, en la "Advertencia" que incorpora a su versión del tratado, pone de manifiesto la importancia de tener una terminología científica en español y su decisión de asignar "terminación castellana" a las voces especializadas.

Palabras clave: Georges Cuvier; José Garriga y Baucís; historia de la traducción científica; enseñanza de la historia natural; terminología científica.

Abstract: This paper aims at analysing José Garriga y Baucís's Spanish version of Tableau élémentaire de l'histoire naturelle des animaux (1797-1798) written by Georges Cuvier (1769-1832), promoter of palaeontology and comparative anatomy. This translation, published in 1834 in Valencia by Cabrerizo's publishing house, is key in marking the arrival of Cuvier's work in Spain, whose main contribution in the development of

》 De Beni, Matteo. 2019. "Georges Cuvier en España. La traducción de José Garriga y Baucís de Lecciones elementales de la historia natural de los animales". Quaderns de Filologia: Estudis Lingüistics XXIV: 309-328. doi: 10.7203/QF.24.16314 
biology and anatomy specifically entails the description - based on structuralist criteriaof the animal kingdom.

The article focuses on aspects that link the text at hand with its "external history" -particularly, its didactic intention in the framework of the current regulations in the public education system of the time- and on the comparison between Cuvier's work and its Spanish translation. This allows for considerations on the peculiarities of the latter with respect to the original text, in order to emphasize the lexical choices and linguistic sensitivity of the translator, who -in the "Advertencia" of his version of the treatise- highlights the importance of having a scientific terminology in Spanish and his consequent decision to assign "terminación castellana" to scientific lexis.

Keywords: Georges Cuvier; José Garriga y Baucís; history of scientific translation; natural history teaching; natural sciences terminology. 


\section{Introducción}

Georges Cuvier (1769-1832) es, sin lugar a duda, un prócer de la ciencia europea entre el periclitar del siglo XVIII y el primer tercio del XIX, gracias a su papel de promotor de la paleontología y de la anatomía comparada. Asimismo, Cuvier contribuyó al debate científico apadrinando teorías de gran calado como el catastrofismo y el principio de correlación de las partes ${ }^{1}$.

En cuanto a su relación con España, sobresale su disertación sobre los famosos restos fósiles de megaterio - una especie de perezoso terrestre y gigante, ejemplo destacado de la megafauna del Pleistocenoencontrados en 1787 cerca de Buenos Aires, en las orillas del río Luján, y trasladados al Real Gabinete de Historia Natural de Madrid en 1788. Cuvier pudo estudiar y dar el nombre de Megatherium americanum a la especie representada por el ejemplar en cuestión gracias a los dibujos realizados en el Gabinete por Juan Bautista Bru de Ramón, diseñador y disecador de la propia institución matritense, y por el grabador Manuel Navarro ${ }^{2}$. Bru, además, hizo un estudio anatómico de los fósiles del animal y los montó con la postura que todavía hoy se conserva en el Museo Nacional de Ciencias Naturales de Madrid ${ }^{3}$.

Los acontecimientos que rodean el hallazgo del megaterio y el interés científico que despertó marcan un hito en la historia de la ciencia. En palabras de Pelayo (1996: 301), "[1]a primera etapa de la paleontología de vertebrados en Europa occidental terminó en 1787 con el

\footnotetext{
${ }^{1}$ A la notoriedad del científico galo contribuyeron, entre otros tratados, Leçons d'anatomie comparée (1. a ed. 1800-1805, 2. . ed. 1835-1845) -que es “[e]l más importante de los textos anatómicos de Cuvier"- y Le régne animal (1817), "su más importante obra de zoología sistemática y quizá la más influyente de toda su producción científica a través de numerosas reediciones y traducciones" (López Piñero, 1992: 22). Como apunta Pimentel, precisamente algunos de los principios fundamentales de las teorías de Cuvier -"su defensa de la Creación, el catastrofismo y la estabilidad de las especies"- acabaron por lastrar "su legado de manera inevitable" (2010: 261).

2 Tradicionalmente las láminas se han atribuido a Bru, pero se trata de un asunto dudoso. Al respecto, véase Pimentel (2010: 150 y 172-179).

${ }^{3}$ Como se explica en la web del Museo, "El megaterio se convierte así en el primer vertebrado fósil de la historia que se montaba con la postura que supuestamente tendría en vida al objeto de exhibirlo públicamente" (Museo Nacional de Ciencias Naturales, 2018). Aunque se sabe que el animal no tiene la postura correcta, el montaje se mantiene en su estado original debido a su valor histórico.
} 
descubrimiento del esqueleto completo del megaterio y su posterior estudio anatómico y determinación, realizado[s] por Cuvier". De hecho, el megaterio le sirvió al anatomista y zoólogo francés para emprender el esbozo de su teoría catastrofista, apuntalada en los fósiles de géneros extinguidos de vertebrados: nada más adecuado para corroborar la idea de una historia de la Tierra marcada por sucesivas hecatombes que encontrar restos de especies de grandes animales totalmente desaparecidas ${ }^{4}$.

En España el estudio anatómico del megaterio llevado a cabo por Bru -con el aparato iconográfico realizado por él y Navarro- y el elaborado por Cuvier vieron los tórculos de la imprenta gracias a José (Joseph) Garriga, "colaborador de Bru en otros proyectos" (Pimentel 2010: 195), quien los reunió en un folleto publicado en 1796 por uno de los más importantes talleres tipográficos de la época, el de Joaquín Ibarra, en aquel entonces en manos de su viuda. El estudio de Bru había permanecido, hasta aquel entonces, inédito, mientras que el de Cuvier se había publicado en el mismo año 1796 y Garriga se encargó de su traducción del francés ${ }^{5}$.

Ahora bien, casi cuatro décadas después, en 1834, la imprenta valenciana de Cabrerizo publicará la traducción al español del tratado Tableau élémentaire de l'histoire naturelle des animaux (1797-1798) de Cuvier bajo el título de Lecciones elementales de la historia natural

\footnotetext{
${ }^{4}$ Además de Pelayo (1996: 290-302) y de Pimentel (2010), sobre la historia de los fósiles del megaterio de Luján, nada exenta de detalles y anécdotas, véase, entre otros, López Piñero \& Glick (1993) y López Piñero (1989).

${ }^{5}$ Antes de la publicación del trabajo de Cuvier, en 1796 en París los fósiles del misterioso animal fueron objeto de dos memorias, leídas en el Institut de France (sesiones del 31 de marzo y del 10 de abril), la primera por Henri Grégoire y la segunda por el propio Cuvier (Pelayo, 1996: 297). En el título del estudio publicado por el científico francés se lee "Notice [...] rédigée par G. Cuvier" (Cuvier, 1796: 303), que el traductor español traduce con "Noticia que da Gregorio Cuvier [...]" (Garriga, 1796: 17, cursiva nuestra). En su Prólogo (s. p.), Garriga se refiere al anatomista y paleontólogo galo como «Gregorio Couvier» $[\mathrm{sic}]$. Es posible que el descuido onomástico de nuestro traductor se deba a su falta de conocimiento, en aquel entonces, de la figura de Cuvier, pero tampoco podemos descartar que el error se haya producido por solapamiento y confusión de los nombres de Grégoire y del propio Cuvier. Fue Henri Grégoire quien en Francia recibió las pruebas de las planchas del megaterio realizadas en el Real Gabinete de Madrid (Pelayo, 1996: 297-298).
} 
de los animales ${ }^{6}$. La versión castellana la firma, como traductor, José Garriga y Baucís.

El objetivo del presente trabajo es abordar el estudio de dicha obra tanto desde una perspectiva "externa" -relacionando la traducción del tratado que nos interesa con las circunstancias y la coyuntura cultural en las que esta se realiza-, como desde una perspectiva "interna", esto es, atendiendo a su dimensión textual, gracias al cotejo del texto meta con el texto fuente.

Más en concreto, dilucidaremos cómo la traducción estudiada entronca con el conjunto de obras de Garriga y con los textos impresos por el taller de Cabrerizo; asimismo, pondremos de manifiesto qué puesto ocupa la obra en cuestión en el panorama de las traducciones del francés de tratados de naturalis historia que se realizan en el segundo tercio del siglo XIX. Por último, llevaremos a cabo un examen textual de la obra, atendiendo a sus elementos textuales y paratextuales y a aspectos de carácter lingüístico.

\section{El traductor y el impresor}

Cabe preguntarse, en primer lugar, si los dos Garriga son la misma persona o si se trata de un caso de (cuasi)homonimia. En la portada del folleto sobre el megaterio se consigna: "publícala don Joseph Garriga, capitan de Ingenieros Cosmógrafos de Estado", mientras que en la traducción de 1834 consta que la versión española es fruto de la pluma de "D. José Garriga y Baucis, Individuo de la Real Academia de la Historia de Madrid, de la de Ciencias naturales i Artes de Barcelona, i de varios otros cuerpos literarios".

Según la Real Academia de la Historia, José Garriga (Barcelona, 1765-?) ingresó en 1804 como supernumerario en dicha corporación, pero de 1814 a 1833 perdió su posición por afrancesado y de 1814 a 1820 vivió exiliado en Francia (López Tabar, 2018). Este dato es coherente con la portada de la traducción de Cuvier de 1834, en la que se firma como "Individuo de la Real Academia de la Historia de Madrid".

\footnotetext{
${ }^{6}$ La portada del texto original consigna la fecha de publicación según el calendario de la Revolución francesa: "an 6", que corresponde a un año entre septiembre de 1797 y septiembre de 1798.

${ }^{7}$ En las citas mantenemos las grafías de los originales.
} 
Se trata de una figura polifacética: profesor, fundador de un importante instituto de enseñanza en la Villa y Corte y jurista, al mismo tiempo que persona interesada por las ciencias, al punto de obtener en 1787 "el título de profesor de Química y de Botánica" y de enseñar "hasta 1797 Matemáticas y Meteorología en el Real Observatorio de Astronomía, y sirvió como capitán de Ingenieros Cosmógrafos" (López Tabar, 2018) ${ }^{8}$. Este último título es el mismo que esgrime la portada de la Descripción de 1796, ya mencionado. Dicho elemento, junto con el hecho de que sabemos que el supernumerario de la Academia de la Historia nació en 1765 y vivió hasta (por lo menos) los años treinta del siglo XIX, demuestra que el José Garriga que edita el folleto de 1796 y José Garriga y Baucís son la misma persona ${ }^{9}$. Así pues, nuestro traductor volvió a trasladar al español un texto de Cuvier casi cuarenta años después de la primera traducción y dos después del óbito del científico francés.

Las dos traducciones en cuestión no son las únicas publicaciones de Garriga, que es un verdadero polígrafo:

Como jurista publicó unas Observaciones sobre El Espiritu de las Leyes y una Continuación y suplemento del Prontuario de Don Severo Aguirre... en siete volúmenes. Ya como profesor, publicó una Gramática de la lengua castellana compuesta por la Academia Española, ordenada en forma de diálogo y aumentada con un tratado de ortografía. Fue autor de varios textos científicos como la Uranografía, o descripción del cielo, un Curso elemental de meteorología, un Discurso sobre la necesidad y utilidad del estudio de la Meteorología y una Cronología teórica y práctica, dedicada al ministro Calomarde (López Tabar, 2018) ${ }^{10}$.

\footnotetext{
${ }^{8}$ Los datos señalados son coherentes con la escueta semblanza del autor que ofrece Aguilar Piñal: "N. en Barcelona (1765). Profesor de matemáticas del Observatorio astronómico de Madrid. Capitán de ingenieros. Alcalde del Crimen de la Audiencia de Galicia" (1981-2001: IV, 170). Es la misma información biográfica que se reproduce en Riera Palmero y Riera Climent (2003: 94).

${ }^{9}$ En cambio, no hay que confundir a José Garriga con otro personaje coetáneo y de nombre semejante: Josep Garriga i Buach, coautor con José María San Cristóbal de Curso de química general aplicada á las artes (1804-1805).

${ }^{10}$ En realidad, también las Observaciones sobre El Espíritu de las Leyes (Madrid, 1787) señaladas en la semblanza citada son el fruto de una traducción del francés. De hecho, como hemos podido comprobar cotejando el texto español con distintas publicaciones que comentan la famosa obra de Montesquieu, se trata de una versión de Observations sur l'Esprit des loix, ou L'art de lire ce livre, de l'entendre et d'en juger, de Joseph
} 
La labor traductora de José Garriga se revitaliza en los años 30 del XIX, cuando, en un breve periodo de tiempo, se publican distintas traducciones suyas -además de la que es objeto de nuestro trabajo-, todas ellas del francés, y en algunos casos de obras enjundiosas ${ }^{11}$ :

- Charles-François Bailly de Merlieux, Manual completo, teórico y práctico del jardinero ó Arte de hacer, y cultivar toda clase de jardines [...], traducido y añadido por D. José Garriga y Baucis. Madrid: Imprenta de los hijos de doña Catalina Piñuela, 1830 (2 vols. $)^{12}$.

- François-Guillaume Ducray-Duminil, Las veladas de la cabaña, ó lecciones de un padre anciano. Traducidas nuevamente de la $7^{a}$ edicion y adornada con láminas, por D. José Garriga y Baucis. Barcelona: Juan Francisco Piferrer impresor, 1834 (4 t.).

- Alexander Adam, Antigüedades romanas de Alejandro Adam, puestas en castellano por Don José Garriga y Baucis. Valencia: Imprenta de Cabrerizo, 1834 (4 t.) ${ }^{13}$.

- Alexandre Moreau de Jonnès, Estadística de España. Territorio, población, agricultura, industria, comercio, navegación, colonias y rentas. Por Al. Moreau de Jonnés [sic]. Puesta en castellano

de La Porte (Ámsterdam, 1751), que reproduce una selección de artículos del famoso pensador francés.

${ }^{11}$ Aguilar Piñal (1981-2001: IV, 170-171) atribuye a José Garriga trece obras (aunque cuatro son ediciones distintas del Prontuario alfabético y cronológico de Severo Aguirre aumentadas por Garriga a partir de 1799), tanto originales como traducidas, pero entre estas últimas no señala ni la traducción de Cuvier ni ninguna otra de las realizadas en los años treinta. También en Riera Palmero y Riera Climent (2003: 94) solo se mencionan dos traducciones, Observaciones sobre El Espíritu de las Leyes (1787) y Diario de los nuevos descubrimientos (1792-1793), que reúne textos técnico-científicos.

${ }^{12}$ En este caso Garriga no solo tradujo la obra, sino que también insertó unos añadidos que, en su opinión, la hacían más completa, aprovechando obras de sus conterráneos: "Por eso he creido que hacia un servicio al Público poniendo en castellano el Manual completo del Jardinero, no sugetándome siempre á traducirle, sino aprovechándome cuando lo he creido conveniente de las obras que varios Sábios y Profesores españoles han publicado sobre diversos ramos de Agricultura, para hacer este Manual español mas completo que el de la última edicion que acaba de hacer Bailly, que es de la que me he valido" (Garriga, 1830: VI). Seguramente para realizar esta labor Garriga echó mano de su experiencia como profesor de Botánica.

${ }^{13}$ Alexander Adam es un erudito escocés (1741-1809). El examen del texto español que hemos realizado demuestra que también esta versión se llevó a cabo a partir del francés, ya que Garriga traduce unas notas del traductor galo de la obra de Adam. 
por Don José Garriga y Baucis. Valencia: Imprenta de Cabrerizo, 1834.

- Jacques Marquet de Montbreton, Baron de Norvins, Historia de Napoleón, escrita en frances por el Señor Norvins y traducida de la quinta edicion por D. José Garriga y Baucis. Valencia: Imprenta de Cabrerizo, 1835-1836 (12 t. $)^{14}$.

Como se desprende de estos datos bibliográficos, en los años 1834-1836 Garriga entabló una estrecha colaboración con el taller de Cabrerizo. A dicho periodo y a la misma colaboración se adscribe también Lecciones elementales de la historia natural de los animales.

Mariano de Cabrerizo (1785-1868) fue un librero, impresor y editor aragonés asentado en Valencia. Debido a su participación en el bando liberal, sufrió la cárcel y otros agravios, que relata en sus Memorias de las vicisitudes políticas ([1854] 1862). Impulsó la publicación en España de novelas extranjeras: alemanas, inglesas y sobre todo francesas, convirtiéndose en promotor de la difusión de autores de gran calado, como Chateaubriand ${ }^{15}$. Además, entre 1830 -cuando "montó imprenta propia" (Alonso, 2017) - y los años cincuenta, Cabrerizo fue el impresor de cuantiosos tratados de distintas disciplinas no literarias: entre otras, de arte de la guerra y táctica militar, de derecho, de medicina, de ámbitos técnico-prácticos (por ejemplo, sobre la cultivación del olivo, la edificación, la cría de los gusanos de seda), entre los cuales caben bien textos originales españoles, bien traducciones, casi todas del francés ${ }^{16}$. En cuanto a la historia natural, no fue un terreno muy transitado

\footnotetext{
${ }^{14}$ El tomo duodécimo de esta obra monumental, también traducido por Garriga, corresponde a Vida privada de la emperatriz Josefina, su familia y su Corte, para conocer mejor al emperador Napoleón y varios hechos de su historia sacados de las memorias de la Sra. Avrillion, camarera de la Emperatriz, que a veces en los catálogos bibliográficos se registra como una obra independiente.

${ }^{15}$ Sobre la trayectoria política y editorial de Cabrerizo, véase Rodrigo Mancho (2018), donde se ofrece abundante información bibliográfica sobre este personaje y datos acerca de las colecciones de novelas que promovió.

${ }^{16}$ En algunos casos la contribución de Cabrerizo a la difusión de tratados científicos franceses no implica su faceta de impresor, pero sí la de editor y librero. En sus memorias, Cabrerizo recuerda como "hallazgo feliz" el haber encontrado La Medicina curativa de Mr. Le Roy, del que dice: "[lo] publiqué en castellano, habiendo vendido en tres años mas de 46000 ejemplares, incluso el tomo de sus Casos prácticos; despacho fabuloso, y de que aun no existia ejemplo en la imprenta española [...]". ([1854] 1862: 136). El tratado en cuestión tuvo un gran éxito y fue impreso por distintos talleres ti-
} 
por la imprenta de Cabrerizo, siendo la traducción de Cuvier la única monografía que hemos encontrado sobre dicho ámbito publicada por su taller. De todos modos, al escudriñar el panorama editorial de la época se observa que la traducción en cuestión no es un caso aislado.

\section{Manuales de historia natural, traducciones del francés y enseñanza}

En el siglo XIX la historia natural entra en una etapa de renovada ebullición. De hecho, se afianzan nuevas teorías sobre el origen y desarrollo del globo y de los seres vivientes, algunas de ellas apuntaladas en rasgos morfológicos y anatómicos que dejan vislumbrar una renovación de los estudios biológicos. La aclimatación en España de dichas teorías es a menudo deudora de autores y textos franceses ${ }^{17}$.

De ahí que, como es fácil de suponer, el tratado traducido por Garriga no fuera una rara avis: entre los años treinta y cuarenta del siglo XIX, también al calor del cambio de régimen que supone el fin del reinado de Fernando VII, se van publicando tratados que sufragan el afianzamiento del estudio de las ciencias naturales, a menudo de origen foráneo ${ }^{18}$. Entre las distintas ediciones de manuales y disertaciones sobre ciencias naturales de autores galos caben, entre otros, Nuevos elementos de historia natural (1837 y 1843) de Antoine Salacroux ${ }^{19}$, Elementos de

pográficos, como el de José Ferrer de Orga (1827), a pesar de que era Cabrerizo quien se encargaba de su venta. En aquel entonces nuestro personaje no contaba con una imprenta propia, pero ya trabajaba como librero y comerciante de libros.

${ }^{17}$ Distintos especialistas han incidido en el relevante papel de las traducciones técnicocientíficas del francés al español; véase, entre otros, Gutiérrez Cuadrado (2004).

${ }^{18}$ Naturalmente no faltan, antes o durante las décadas señaladas, obras de autores españoles de historia natural: baste mencionar el caso de Agustín Yáñez y Girona, quien, en su Lecciones de Historia natural abarca zoología, botánica y mineralogía (1. a ed. 1820; 2. ${ }^{a}$ ed. 1844-1845); en cuanto a la zoología, Cuvier es un punto de referencia irrenunciable para Yáñez, en particular para la segunda edición de su manual, aunque luego se acercó a las ideas de Lamarck (véase al respecto Sucarrats i Riera, 2006). Además, en la época señalada también se reeditan obras clásicas de las ciencias naturales, como es el caso de los compendios ad usum Delphini del célebre Comte de Buffon (El Buffon de los niños, ó historia natural abreviada de los cuadrúpedos, aves, anfibios, insectos, etc., 1834).

19 Pinilla Martínez (2016) estudia las distintas traducciones del tratado de Salacroux al español, la primera realizada en 1837, las otras dos en 1843. La primera edición del texto fuente es de 1836 . 
zoología, o Historia natural de los animales (1843) de Milne-Edwards y Achille Comte ${ }^{20}$ o Curso elemental de historia natural. Mineralogía (1847) de François Sulpice Beudant ${ }^{21}$.

En los tres casos señalados no se trata de traducciones tardías como pasa con la de Cuvier-Garriga, donde más de treintaicinco años separan el texto fuente de su edición española. La fama de Cuvier convierte a su obra en un clásico de la historia natural y este hecho de por sí solo justifica que se traduzca al español:

El nombre de Cuvier es tan famoso en Europa por los grandes adelantamientos que ha hecho en la Historia natural, que se buscan con ansia las ediciones de las obras del inmortal conde de Buffon, por las adiciones i complemento que han recibido sacados de las de Cuvier. No se necesita mas que esto para juzgar el justo aprecio que han merecido en todas partes las Lecciones que publicamos (Garriga, "Advertencia del Traductor" en Cuvier, 1834: s. p., cursiva en el texto).

Además de la fama de Cuvier, es preciso poner el foco en un aspecto que aúna este tratado con las demás traducciones mencionadas: su potencialidad como textos docentes.

Distintos testimonios nos recuerdan el papel que desempeñaron en las décadas en cuestión las comisiones de instrucción pública y la escasez de libros adecuados en español para las asignaturas de carácter científico. Por ejemplo, la Comisión de examen de libros en su relación de textos recomendados del 8 de octubre de 1841 escribe lo siguiente sobre la obra de Salacroux que hemos mencionado:

Con respecto a esta asignatura dice la sección que mediante la escasez de obras elementales puestas en castellano, merecen en concepto de sus individuos cierto aprecio los nuevos elementos de Salacroux traducidos por D. José Rodrigo, aunque para obra de asignatura para una escuela de Historia natural les parece demasiado, y como clásica no consideran que presente ventajas a la de Cuvier, Cavanilles, Lineo y otros muchos

\footnotetext{
${ }^{20}$ La primera edición del texto fuente es de 1833. En la portada de la traducción española se dice que el traductor, Pedro Barinaga, realizó su labor a partir de la cuarta edición. ${ }^{21}$ El original francés es de 1841 y formaba parte de un curso completo de Historia natural en el que Henri Milne-Edwards se encargó de la parte de zoología y Adrien de Jussieu de la de botánica. No hemos podido localizar y consultar traducciones al español de estas otras dos partes.
} 
autores de que necesariamente tienen que valerse los profesores, causa por la cual no puede señalarse ninguna obra como exclusiva para estas asignaturas especiales (Villalaín, 1997: II, 24. Apud Pinilla Martínez, 2016: 269).

La obra de Salacroux no es el único caso en la época acotada de traducción de un tratado francés de historia natural que está estrechamente vinculado con el contexto de la enseñanza. Ni mucho menos. Ciñéndonos a los textos mencionados supra, la portada del de Beudant (1847) asevera que el texto fue "[a]doptado para la enseñanza en Francia para el Consejo Real de Instruccion Pública", mientras que en la del tratado de Milne-Edwards y Comte (1843) se pone de manifiesto que se escribió "en frances para uso de los colegios y de las escuelas normales", recalcando además que es

[o]bra adoptada por el Consejo real de Instruccion pública para servir de testo en la enseñanza de la Historia natural en los Establecimientos de la Universidad de Francia.

En cuanto al caso aquí estudiado, el vínculo con la esfera didáctica se colige ya del título escogido para la traducción española del tratado de Cuvier: Lecciones elementales [...] dadas por G. Cuvier en la Escuela del Panteon de Paris ${ }^{22}$. Aunque el título en castellano no reproduce literalmente el de la obra original francesa (Tableau élémentaire), mantiene su intención instructiva y sinóptica. Según una de las definiciones del Dictionnaire de l'Académie française (6. ${ }^{\mathrm{a}} \mathrm{ed}$.), la voz tableau

[...] se dit également d'une feuille, d'une planche sur laquelle des matières didactiques, historiques et autres, sont rédigées et rangées méthodiquement, pour être vues d'un coup d'oeil (Institut de France, 1835).

No es casualidad que tableau, a menudo acompañado por élémentaire para señalar que la materia tratada se ha reducido a sus principios básicos, sea el atributo que prima en las portadas de muchos tratados franceses de finales del siglo XVIII y de la centuria siguiente.

\footnotetext{
${ }^{22}$ Además, en la anteportada, que solo consigna el título resumido y la indicación del tomo (primero o segundo), se lee: Elementos de la historia natural de los animales.
} 
Además, cabe destacar que, en el prefacio escrito por Cuvier, traducido fielmente por Garriga, se hace alarde de la introducción de la historia natural como disciplina de la enseñanza reglada:

El estudio de la Historia natural, que no se comprendia en el antiguo sistema de enseñanza pública, se ha substituido actualmente a otros conocimientos menos útiles, $i$ esto ha hecho sensible la necesidad de unos elementos que presentasen tanto a los maestros como a los discípulos en compendio, pero con solidez, el verdadero estado de la ciencia en la actualidad, i esta es la mira que me ha decidido a publicar en resúmen las lecciones que di en la escuela del Panteon (Cuvier, 1834: vii).

Desde la imprenta de Cabrerizo, editor e impresor de la traducción de Garriga, al promover la publicación de la obra de Cuvier se incide no solo en la fama del autor francés, sino también en la necesidad de obras que, como esta, puedan servir para paliar la escasez de textos docentes de historia natural. Es lo que se desprende del anuncio insertado al final del tomo I de la monumental Historia de Napoleón, publicada por Cabrerizo en traducción del propio Garriga y Baucís:

[...]. Esta obra elemental [el tratado de Cuvier], clásica en la parte de historia natural de los Animales, no podrá menos de ser buscada entre nosotros, porque carecemos todavía de elementos de esta ciencia, la cual vemos difundirse con velocidad desde que se ha conocido su importancia, y estamos seguros que debe ocupar su lugar en un plan de estudios arreglado á las luces del siglo (Anuncio en el tomo I de Marquet de Montbreton, 1835: s. p. $)^{23}$.

El científico francés ocupa un lugar destacado en el ámbito de la enseñanza de la historia natural, en el que "la classificació de Cuvier es va imposar sense discussió, com a mínim fins a mitjans de segle". De hecho, tanto en Francia como en el resto de Europa, "[e]l sistema de

\footnotetext{
${ }^{23}$ Esta información de carácter promocional se hace eco de las palabras empleadas por Garriga para introducir el texto español. Confróntese con esta cita con el fragmento de la Advertencia del Traductor reproducido supra y con el que sigue: "Esta obra elemental es clásica en la parte de Historia natural de los Animales, que es la que trata, i no puede menos de ser mui apreciada entre nosotros que carecemos de Elementos de esta Ciencia, que va difundiéndose con velocidad desde que se conoce mas su importancia, $i$ desde que el Gobierno con su protectora mano la fomenta" (Garriga, Advertencia del Traductor en Cuvier, 1834: s. p., cursiva en el texto).
} 
Cuvier era seguit de forma majoritària pels autors de llibres de text, i en general pels zoòlegs" (Sucarrats i Riera, 2006: 143 y 220).

También es significativo, en el contexto de la época, el llamamiento a la necesidad de reformas educativas que abarquen la historia natural. Al año siguiente, en 1836, se aprobó el Plan General de Instrucción Pública ("Plan Rivas"), que introducía elementos de ciencias naturales en la instrucción secundaria, pero asimismo en la "instrucción primaria superior", en la que incluía "Nociones generales de física, química e historia natural, acomodadas a las necesidades más comunes de la vida". Las normas de dicho Real Decreto quedaron en papel mojado debido a desavenencias políticas, pero estos atisbos se afianzaron con la legislación siguiente, como el "Plan Pidal" (1845) y la Ley de Instrucción Pública, conocida como "Ley Moyano" (1857). De ahí que algunos textos se plasmaran para cumplir los requerimientos de los planes de enseñanza reglada vigentes, como en el caso de Curso metódico de nociones de Historia natural popular. Al uso de las escuelas primarias, secundarias y normales (1843), de José María Paniagua, incluido en el catálogo de los libros de texto para la instrucción primaria elemental con la Real Orden del 30 de junio de 1848, la cual advertía: "se usen esclusivamente las obras comprendidas en el adjunto catálogo" (Colección de reales decretos, órdenes y reglamentos, 1850: 248).

\section{Aspectos macro-y microtextuales}

En cuanto a la caracterización física de la traducción, hay que señalar que difiere en parte de la de su texto fuente. Este último se publicó en un único tomo (710 pp.; más otras 16 , señaladas con números romanos, que corresponden a anteportada, portada, prefacio y explicación de las láminas). En cambio, la traducción se reparte en dos tomos (de 358 y 394 pp., respectivamente, más 16 que incluyen los mismos elementos paratextuales del texto fuente, además de una breve Advertencia del Traductor).

Por lo que se refiere a la organización de los contenidos, Cuvier, después de la introducción, vertebra la obra en ocho libros dedicados a sendos temas: hombre (entendido naturalmente como ser humano), mamíferos, aves, réptiles (que incluyen también los anfibios), peces, moluscos, insectos y gusanos, zoófitos. En la traducción se respeta la misma división en libros, pero los últimos tres, que describen los 
invertebrados, conforman el segundo tomo, así que el primero se consagra a los vertebrados. Además, en la traducción se reproducen las 14 láminas del texto fuente, aunque en el texto meta se incluyen 7 en cada tomo; tal distribución equitativa corresponde a la repartición de la materia tratada en cada uno de los volúmenes (vertebrados frente a invertebrados) $)^{24}$.

En la Advertencia del Traductor, Garriga asevera haberse ajustado a «la actual ortografía castellana» (s. p., cursiva en el texto), en concreto haber evitado rasgos en desuso y meramente etimológicos. Es un claro indicio de su especial atención a los aspectos lingüísticos, incluso a los formales y ortográficos ${ }^{25}$. Asimismo, asevera no haberse apartado del texto fuente -lo cual es cierto-, pero al mismo tiempo reivindica la castellanización de la nomenclatura zoológica:

En la traduccion no me he apartado del autor, porque seria mucha presuncion querer correjir a tal maestro; pero he cuidado de dar terminacion castellana a varios nombres que algunos han usado con las estranjeras; he adoptado la traduccion o terminacion castellana tomada de la nomenclatura latina cuando he dudado de la lejitimidad de la adopcion de alguna voz [...]. (s. p., cursiva en el texto)

Algunas de las elecciones lingüísticas de Garriga frente a las de Cuvier merecen especial atención, aunque tan solo daremos algunas pinceladas, puesto que un análisis terminológico completo rebasa los límites del presente artículo.

\footnotetext{
${ }^{24}$ En cuanto a la estructura del tratado, añadimos que al final del mismo, bien en francés bien en español, se encuentran dos índices terminológicos: uno registra en orden alfabético los nombres de animales en lengua vernácula empleados en el manual ("Table de noms français, des classes, des ordres, des genres, et de leurs subdivisions" / "Índice castellano con la correspondencia latina"), el otro, en cambio, reúne los nombres latinos ("Table de noms latins" / "Tabla de los nombres jenéricos latinos"). Ambos índices reenvían al lector a la página en la que aparece cada nombre. El índice de nombres vernáculos realizado por Garriga es más amplio que el de Cuvier, porque el español acompaña cada zoónimo en castellano con su correspondiente nombre científico.

${ }^{25}$ Cabe recordar, como síntoma más de su interés por la lengua, que en su heterogénea obra se encuentra una Gramática de la lengua castellana compuesta por la Academia española, ordenada en forma de diálogo y aumentada con un tratado de ortografía (1823). El título sugiere que se trata de uno de los numerosos compendios de la gramática académica que se fueron realizando y que, además, emplea el método erotemático, según una tradición gramatical bien asentada.
} 
Garriga se decanta por adaptar algunos elementos del texto fuente para acercarlos a un lector conterráneo. Es el caso de la sustitución de citoyen, voz fuertemente arraigada en el contexto de la Revolución francesa en el que Cuvier vivió, por un más neutro señor. De ahí que, por poner uno de entre los muchos ejemplos encontrados, "le citoyen Lamarck" pase a ser "el señor Lamarck" (Cuvier, 1797-1798 y 1834: ix, cursivas en los textos). Otro ejemplo es la traducción de "collection national" por "gabinete":

Toutes les indications de dents ou d'autres caractères, que l'on trouvera différentes de celles données par les auteurs connus, ont été prises d'après nature sur les individus de la collection nationale, et on peut $\mathrm{y}$ avoir une entière confiance (1797-1798: vi).

Todo lo que toca a los dientes o a otros caractéres distinto de lo que han dicho los autores conocidos, se ha tomado de los animales mismos que existen en el Gabinete, $i$ asi se debe tener entera confianza en ello (1834: viii).

Sin duda, Garriga tiene el referente del Real Gabinete de Historia Natural de Madrid, con el que -como hemos visto- entró en contacto, aunque hay que añadir que en 1815 ya existía en la Villa y Corte el Real Museo de Ciencias Naturales ${ }^{26}$, que aprovechó los fondos del antiguo Gabinete. La denominación de museo frente a gabinete se fue afianzando en España en el siglo XIX, coherentemente con lo que pasaba en Europa, a medida que las colecciones científicas fueron consolidando su perfil de institución abierta al público general. Un testimonio de estas circunstancias de transición lo proporciona Domínguez en su diccionario (1846-1847) al consignar que la voz museo "se suele usar [...] como equivalente de gabinete".

Otros rasgos, en cambio, no sufren ningún proceso de domesticación en el paso del texto fuente al texto meta: es el caso de algunas referencias de Cuvier a la tesitura histórica y cultural en la que escribe su tratado, como las referidas al calendario francés de la Revolución: "thermidor an 4" (Cuvier 1797-1798: 159, nota) se traduce con "hermidor [sic] del año 4. " (1834: t. I, 144, nota, cursiva en el texto).

\footnotetext{
${ }^{26}$ Desde 1913 denominado Museo Nacional de Ciencias Naturales, su nombre actual.
} 
En cuanto a la descripción de cada animal, esta va encabezada por el zoónimo vulgar, acompañado por el nombre científico entre paréntesis, según la clasificación binomial de cuño linneano. Con respecto al texto fuente, Garriga, en ocasiones, añade otra denominación vernácula, sin duda para facilitar a su lector la identificación del animal en cuestión. Es así como el apartado dedicado a "Les tatous" (1797-1798: 129) pasa a titularse "Los tatus o armadillos" (I, 144), o como "le chamois (Antilope rupicapra)" (1797-1798: 163), además de "gamuza", recoge la denominación española "cabra montés" (1834: I, 147). También en "la manucode (Paradisea regia)" (1797-1798: 209) se encuentra variación denominativa: "ave real del paraíso o la munucodiata [sic]" (1834: I, 193). A veces este procedimiento sinonímico puede llevar a cierta confusión. Este es, por ejemplo, el caso observado en el tratamiento de los tiburones (1797-1798: 316-317 y 1834: I, 302). En ambas versiones se menciona el Squalus carcharias, nombre científico por aquel entonces en uso, acuñado por Linneo para clasificar el tiburón blanco. Cuvier emplea el nombre vulgar de "requin" y Garriga el de "tiburón", los dos muy generales. Pero Garriga, en la descripción del animal, introduce un añadido pensando en su lector: "Algunos le llaman Lija". Más abajo, para denominar otro escualo, en concreto, "la roussette (Squalus canicula)" según la denominación de Cuvier, Garriga emplea de nuevo el término "la lija (Squalus canicula)". Así pues, los zoónimos vernáculos resultan muy útiles para el lector lego, pero al mismo tiempo conllevan falta de precisión terminológica o, incluso, círculos viciosos denominativos, en los que un nombre vulgar corresponde a dos seres vivos distintos.

\section{Conclusiones}

La traducción de Lecciones elementales de la historia natural de los animales de Georges Cuvier realizada por José Garriga y Baucís es un testimonio privilegiado para el estudio de la lengua de la zoología en la primera mitad del siglo XIX. Esto se debe a que concurren distintas circunstancias.

En primer lugar, el hecho de que su autor es una figura imprescindible de los estudios zoológicos en la época y que la labor de su traductor no es ajena a reflexiones metalingüísticas que le hacen tomar partido a favor de la castellanización de la nomenclatura zoológica y asimis- 
mo -como se desprende del cotejo del texto fuente con el texto metale estimulan a enriquecer su traducción con zoónimos vernáculos que amplifican el texto original. Esta variación denominativa, como hemos visto con el ejemplo de la voz lija, desemboca en ocasiones en la generalización, entorpeciendo la identificación de una especie animal con respecto a otra: las denominaciones vulgares, más cómodas y cercanas para un lector lego, resultan a veces tramposas debido a su carácter poco especializado. Para evaluar el trabajo de traducción de Garriga hay que considerar, empero, las dificultades que entraña la descripción naturalista $-\mathrm{y}$, por ende, el denominar una especie- en una época en la que todavía el vocabulario de la zoología en español necesitaba afianzarse.

En segundo lugar, hay que recalcar que el tratado objeto de estudio se publica en un momento de efervescencia de los estudios sobre la historia natural y su naturaleza textual -esto es, el hecho de que es una traducción de un texto divulgativo y pensado para la enseñanza- no invalida sus potencialidades para el estudio del vocabulario de la zoología de la primera mitad del siglo XIX. Todo lo contrario: las traducciones, en especial las realizadas a partir del francés, fueron un medio de transmisión de ideas científicas y de las voces relacionadas con estas. En cuanto a los manuales para la enseñanza, los textos docentes y de carácter divulgativo son fuentes importantes para el estudio diacrónico del léxico, ya que aportan indicios sobre la difusión de los términos, su alcance y frecuencia de uso.

En este trabajo nos hemos dedicado con cierto detenimiento a situar el texto en su contexto. De hecho, creemos que para examinar un vocabulario de especialidad es provechoso escudriñar las obras que fueron su vehículo de difusión y que no se puede desligar el estudio histórico del léxico de las circunstancias en las que este empezó a circular. Por consiguiente, a partir de este trabajo, esperamos poder estudiar en un futuro de forma sistemática la andadura de las voces de la zoología empleadas por Garriga en su traducción bien en otras obras del ámbito de la historia natural, bien en la lexicografía del español.

\section{Bibliografía}

Aguilar Piñal, Francisco. 1981-2001. Bibliografía de autores españoles del s. XVIII. Madrid, CSIC (10 vols.). 
Beudant, François Sulpice. 1847. Curso elemental de historia natural. Mineralogía [...] Traducción de don Cayetano Balseyro. Madrid: Imprenta de la Viuda de Jordán e Hijos.

Cabrerizo, Mariano. [1854] 1862. Memorias de las vicisitudes políticas de D. Mariano de Cabrerizo y Bascuas (2. ${ }^{\text {a }}$ ed.). Valencia: Imprenta de los señores Ferrer y Aisa, antes de Cabrerizo.

Colección de reales decretos, órdenes y reglamentos. (1850). Colección de reales decretos, órdenes y reglamentos relativos a la instrucción primaria, elemental y superior. Madrid: Imprenta la v. de Perinat y Compañía.

Cuvier, Georges. 1796. Notice sur le squelette d'une très grande espèce de quadrupède inconnue jusqu'à présent, trouvé au Paraguay, et déposé au cabinet d'Histoire Naturelle de Madrid, rédigée par G. Cuvier. Magasin Encyclopédique ou Journal des Sciences, des Lettres et des Arts année 2(1): 303-310.

Cuvier, Georges. 1797-1798. Tableau élémentaire de l'histoire naturelle des animaux. Paris: Baudouin.

Cuvier, Georges. 1834. Lecciones elementales de la historia natural de los animales, dadas por G. Cuvier en la Escuela del Panteon de París $i$ traducidas por don José Garriga y Baucis. Valencia: Imprenta de Cabrerizo (2t.).

Domínguez, Ramón Joaquín. 1846-1847. Diccionario nacional o gran diccionario clásico de la lengua española. Madrid: Bernat.

Garriga y Baucís, José. 1830. Prólogo. En Bailly de Merlieux, CharlesFrançois, Manual completo, teórico y práctico del jardinero ó Arte de hacer, y cultivar toda clase de jardines [...]. Madrid: Imprenta de los hijos de doña Catalina Piñuela, vol. I, III-VI.

Garriga, Joseph (1796) (ed.). Descripcion del esqueleto de un quadrúpedo muy corpulento y raro, que se conserva en el Real Gabinete de Historia Natural de Madrid. Madrid: En la imprenta de la viuda de don Joaquin Ibarra.

Gutiérrez Cuadrado, Juan. 2004. Las traducciones francesas, mediadoras entre España y Europa en la lengua técnica del siglo XIX. En Alsina, Victoria; Brumme, Jenny; Garriga, Cecilio \& Carsten, Sinner (ed.) Traducción y estandarización. La incidencia de la traducción en la historia de los lenguajes especializados. Madrid: Vervuert / Iberoamericana, 35-60.

Institut de France. 1835. Dictionnaire de l'Académie française (6. ${ }^{\mathrm{a}}$ ed.). Paris: Firmin-Didot frères. https://artfl-project.uchicago.edu/content/ dictionnaires-dautrefois [Acceso 15/04/2019].

López Piñero, José María \& Glick, Thomas F. 1993. El megaterio de Bru y el Presidente Jefferson. Una relación insospechada en los albores de la 
paleontología. Valencia: Instituto de Estudios Documentales e Históricos sobre la Ciencia.

López Piñero, José María. 1989. Juan Bautista Bru y la difusión por Cuvier de su obra paleontológica. Arbor 120(14): 79-99.

López Piñero, José María. 1992. La anatomía comparada antes y después del darwinismo. Madrid: Akal (Historia de la ciencia y de la técnica, 41).

López Tabar, Juan. 2018. José Garriga. Real Academia de la Historia. http:// dbe.rah.es/biografias/83642/jose-garriga [Acceso 08/03/2019].

Marquet de Montbreton, Jacques, Baron de Norvins. 1835. Historia de Napoleón, escrita en frances por el Señor Norvins y traducida de la quinta edicion por D. José Garriga y Baucis. Valencia: Imprenta de Cabrerizo (12 t.).

Milne-Edwards, Henri \& Comte, Achille Joseph 1843. Elementos de Zoología, o Historia natural de los animales. Escritos en francés para usos de los colegios y de las escuelas normales. Traducidos al castellano de la cuarta edición por Pedro Barinaga. Madrid: Compañía General de Impresores y Libreros del Reino.

Museo Nacional de Ciencias Naturales. 2018 (16 de mayo). El perezoso gigante que fascinó al mundo. http://www.mncn.csic.es/InformacionGeneralContacto/Blog_El_perezoso_gigante_que_fascino_al_mundo/ seccion $=1235 \&$ idioma $=$ es ES\&id $=2018051612050001 \&$ activo $=12$.do [Acceso 08/03/2019].

Pelayo, Francisco. 1996. Del diluvio al megaterio. Los orígenes de la Paleontología en España. Madrid: CSIC.

Pimentel, Juan. 2010. El Rinoceronte y el Megaterio. Un ensayo de morfología histórica. Madrid: Abada.

Pinilla Martínez, Julia. 2016. Ciencias naturales, enseñanza y traducción. En De Beni, Matteo (ed.) Ciencias y traducción en el mundo hispánico. Mantova: Universitas Studiorum, 263-281.

Plan General de Instrucción Pública. 1936 (4 de agosto). Plan general de Instrucción Pública. Real decreto. ["Plan Rivas"]. http://www.filosofia. org/mfa/fae836a.htm [Acceso 03/04/2019].

Riera Palmero, Juan \& Riera Climent, Luis. 2003. La ciencia extranjera en la España ilustrada. Ensayo de un Diccionario de Traductores. Valladolid: Universidad de Valladolid / Ediciones del Seminario de Historia de la Medicina.

Rodrigo Mancho, Ricardo. 2018. Mariano Cabrerizo: energía literaria y liberal en tiempos de Fernando VII. Cuadernos de Ilustración y Romanticismo 24: 93-114.

Sucarrats i Riera, Raimon. 2006. L'ensenyament de la història natural a la Barcelona de la primera meitat del segle XIX. Els llibres de text $i$ la 
docència d'Agustí Yàñez i Girona (Tesis Doctoral). Bellaterra: Universitat Autònoma de Barcelona / Centre d'Estudis d'Història de les Ciències. [Base de datos Tesis doctorals en Xarxa]. https://www.tesisenred. net/handle/10803/3777.

Villalaín Benito, José Luís. 1997. Manuales escolares en España. Madrid: UNED (3 vols.). 